\title{
Gamification for Volunteer Cloud Computing
}

\author{
Alimohammad Shahri, Mahmood Hosseini, Raian Ali \\ Bournemouth University, UK \\ \{ashahri, mhosseini, rali\}@bournemouth.ac.uk
}

\begin{abstract}
Volunteer cloud computing is a paradigm where idle computing resources owned by a certain user are offered in the cloud to be utilised by others in order to reduce operational costs. Engaging people in volunteer cloud computing is an obstacle which requires novel motivational methods, ideally online. Gamification is a method for increasing people motivation and changing their behaviour towards certain tasks in a nongame context. This position paper advocates that gamification is potentially a means to increase people engagement and retention in volunteer cloud computing projects. As an initial step to reach a systematic application of gamification in such projects, we study the relationship between game mechanics and different categories of volunteers and propose a preliminary mapping that relates these two aspects.
\end{abstract}

Keywords-volunteer cloud computing; gamification mechanics; volunteer classes

\section{INTRODUCTION}

Volunteer cloud computing is the combination of volunteer computing, that is a form of distributed computing technology where resources are offered and shared by people, and cloud computing which offers the platform for that to happen. In volunteer cloud computing, contributors may freely join the cloud platform and share their computing resources with others in the cloud. It has gained success in major platforms such as BOINC [1], SETI@home [2], and ScienceCloud [3].

Gamification, as defined by Deterding et al. [4], is the use of game design elements in a non-game context. The main objective of gamification is to motivate people to engage more with the task at hand. The use of game elements, such as leaderboards and points, is considered to increase the engagement of people who are performing a certain task. For example, game mechanics can be used in call centre to motivate staff to answer customers' calls positively although they may not be genuinely interested in the task. Gamification planning should consider the long term effect, and the personality types, e.g., the same reward may not suffice after a while, or some staff like social recognition while others do not.

Attracting and retaining participants in volunteer computing projects remains an issue, as noted in [5]. Volunteers could leave the platform upon satisfying their initial curiosity. Our position paper proposes the use of gamification in order to engage participants in volunteer computing projects, and to retain their participation and motivate them to stay. Currently, points are used as a reward in such projects but this is too basic and it does not consider the different classes of volunteers and what motivate them. We propose that planning gamification should be adaptive to the volunteers' classes.

\author{
Fabiano Dalpiaz \\ Utrecht University, The Netherlands \\ f.dalpiaz@uu.nl
}

\section{VOLUNTEERS TYPES}

Volunteer computing projects, including those performed in the cloud are typically set up by an assembly of researchers or business executives who recruit willing participants to share their spare computational resources for the processing of computationally intensive models or scientific data. It remains a challenge for researchers to retain the recruited participants as there usually is a very high drop-out rate, as expressed in [5]. The paper continues to suggest recommendations to scientists and researchers in order to enhance volunteer retention rates and classifies the volunteers in three categories:

- Super-crunchers, who generously offer their computers to process a particularly large quantity of project data and expect a good return.

- Lay public, would like the project to succeed but would not sacrifice much resources for it and would not, thus, expect much rewards.

- Alpha-testers, who are selected and invited to test new features in the alpha testing phases and happy to be distinguished to play that role.

\section{GAMIFICATION AND VOLUNTEER CLOUD COMPUTING}

Gamification offers mechanics, such as leaderboards, points, badges, and avatars to motivate and retain volunteers in a business project to further continue with the task at hand. The application of gamification needs careful design to be efficient in leading to increased engagement and trustworthy participation and group work [6]. We advocate that part of that planning for volunteer computing projects is the correct matching between game mechanics and volunteer types. This section studies that relation and proposes a framework that matches the volunteer types to players' types, classified mainly by Bartle's taxonomy of player personalities [8]. A player type is an identifier for the suitable game mechanics. Table 1 summarises our proposal.

Table 1: Volunteer types vs. Gamification

\begin{tabular}{|c|c|c|}
\hline $\begin{array}{c}\text { Volunteer } \\
\text { Types }\end{array}$ & Player Types & $\begin{array}{c}\text { Example Game } \\
\text { Mechanics }\end{array}$ \\
\hline Super-cruncher & $\begin{array}{c}\text { Achievers } \\
\text { Competitors } \\
\text { Non-collaborative } \\
\text { Socializer }\end{array}$ & $\begin{array}{l}\text { Leaderboards } \\
\text { Points } \\
\text { Online forums }\end{array}$ \\
\hline Lay public & $\begin{array}{c}\text { Explorers } \\
\text { Collaborative } \\
\text { Non-competitive } \\
\text { Socializer }\end{array}$ & $\begin{array}{l}\text { Epic meanings } \\
\text { Online forums }\end{array}$ \\
\hline Alpha-testers & $\begin{array}{c}\text { Uniqueness seekers } \\
\text { Socializers } \\
\text { Killers }\end{array}$ & $\begin{array}{c}\text { Badges } \\
\text { Statuses } \\
\text { Avatars }\end{array}$ \\
\hline
\end{tabular}




\section{A. Super-Cruncher}

This class of volunteers constitutes approximately 10 per cent of all active volunteers [5]; super-crunchers usually restrict themselves to a limited number of volunteer computing projects. This allows them to collect a higher number of credits in particular projects. Despite the low percentage, their contribution is significant, forming 60 per cent of all the credits awarded. It is also noted that super-crunchers enjoy the social recognition obtained from high credit scores within the online community of volunteers. Furthermore, they seem to be more enthusiastic in posting in online forums about their particular achievements and they carefully select their projects.

Super-crunchers' eagerness to collect credits suggests that they are more competitive than collaborative, and therefore competitive game mechanics, such as leaderboards and points can further motivate them and retain them within the project. Furthermore, their need for social recognition in online forums can be fulfilled by the use of game mechanics, such as avatars, status and badges, which enhance this standpoint and help, retain their presence in the project.

\section{B. Lay Public}

This class of volunteers form approximately 80 per cent of all active volunteers [5]. However, their contribution is estimated to be only 25 per cent of all the credits awarded. They claim that they are not motivated by the accumulation of credits, and they actually resent the idea of such motivation. They think that they are contributing to science and society by their participation, and accumulating credits trivialises their efforts. While some researches show otherwise [7], it has been suggested that this characteristic type uses credits to reassure their continuing contribution to science and the overall progress of the project.

Lay public characters show an eagerness for collaborative game mechanics rather than competitive ones, such as community collaboration. Furthermore, their willingness to contribute to science and society can be further utilised by game mechanics such as epic meaning (i.e., make the users believe they are working for a great, awe-inspiring enterprise).

\section{Alpha-Testers}

This class of volunteers are recruited by volunteer computing projects at the alpha stage of development, only through an invitation [5]. Like super-crunchers, they enjoy social recognition, but the type of social recognition is different, as they are the only volunteers who get a formal invitation to take part in a project. As a result, they can be highly trusted to retain in a project, and they tend to keep their reputation high to ensure they will get invited again, leading to more social recognition for them. However, since their collected credits are not automatically transferred to their overall credit scores for a particular project as an alpha-tester, they are impervious to the use of credits.

Alpha-testers' high keenness to gain social recognition, which does not happen through accumulation of credits, means that points and leaderboards cannot be applied to them to retain them in the project, as was the case with super-crunchers. Nonetheless, we propose that the use of badges and statuses reflects such social recognition. Furthermore, alpha-testers can enjoy avatars as another non-point-based game mechanic. Alpha testers enjoy the fact that they have been selected while others were not invited. Thus, the Killer player type of Bartle would also fit them.

\section{CONCLUSION AND FUTURE WORK}

In this paper, we have briefly described the different classes of volunteers and their characteristics. We discussed the appropriate types of game mechanics that can be applied to every class of volunteers in a volunteer cloud computing project. This is meant as an initial argument and we propose the conduction of empirical studies to test and validate the hypotheses of this paper. The general research question would be the planning of gamification for a non-profit context and players doing work on a voluntary basis.

\section{ACKNOWLEDGMENT}

The research was supported by an FP7 Marie Curie CIG grant (the SOCIAD Project) and by Bournemouth University through the Fusion Investment Fund (the BBB, BUUU and VolaComp projects) and the Graduate School Santander Grant for PGR Development.

\section{REFERENCES}

[1] D. P. Anderson, "Boinc: A system for public-resource computing and storage," Proceedings of the Fifth IEEE/ACM International Workshop in Grid Computing, 2004, pp. 4-10.

[2] D. P. Anderson, J. Cobb, E. Korpela, M. Lebofsky, and D. Werthimer, "SETI@ home: an experiment in public-resource computing," Commun. ACM, vol. 45, no. 11, 2002, pp. 56-61.

[3] P. Mayer, A. Klarl, R. Hennicker, M. Puviani, F. Tiezzi, R. Pugliese, J. Keznikl, and T. Bure, "The autonomic cloud: a vision of voluntary, peer-2-peer cloud computing," IEEE 7th International Conference on Self-Adaptation and Self-Organizing Systems Workshops (SASOW), 2013, pp. 89-94.

[4] S. Deterding, D. Dixon, R. Khaled, and L. Nacke, "From game design elements to gamefulness: defining gamification," Proceedings of the 15th International Academic MindTrek Conference: Envisioning Future Media Environments, 2011, pp. 9-15.

[5] P. Darch and A. Carusi, "Retaining volunteers in volunteer computing projects," Philos. Trans. R. Soc. A Math. Phys. Eng. Sci., vol. 368, no. 1926, 2010, pp. 4177-4192.

[6] P. Herzig, M, Ameling, A. Schill, "A Generic Platform for Enterprise Gamification," in Software Architecture (WICSA) and European Conference on Software Architecture (ECSA), 2012 Joint Working IEEE/IFIP Conference on. IEEE, 2012, pp. 219-223.

[7] C. Christensen, T. Aina, and D. Stainforth, "The challenge of volunteer computing with lengthy climate model simulations," in e-Science and Grid Computing, 2005. First International Conference on, 2005, pp. 815.

[8] G. Zichermann, and C. Cunningham, Gamification by design: Implementing game mechanics in web and mobile apps, O'Reilly Media, Inc., 2011. 